\title{
Retrosigmoid craniotomy for resection of acoustic neuroma with hearing preservation: a video publication
}

\author{
Jonathan A. Forbes, M.D. ${ }^{1}$, Matthew L. Carlson, M.D. ${ }^{2}$, Saniya S. Godil, M.D. ${ }^{1,}$ \\ Marc L. Bennett, M.D. ${ }^{2}$, George B. Wanna, M.D. ${ }^{2}$, and Kyle D. Weaver, M.D. ${ }^{1}$ \\ Departments of Neurosurgery $y^{l}$ and Otolaryngology ${ }^{2}$, Vanderbilt University Medical Center, Nashville, Tennessee
}

In this publication, video format is utilized to review the operative technique of retrosigmoid craniotomy for resection of acoustic neuroma with attempted hearing preservation. Steps of the operative procedure are reviewed and salient principles and technical nuances useful in minimizing complications and maximizing efficacy are discussed.

The video can be found here: http://youtu.be/PBE5rQ7B0Ls.

(http://thejns.org/doi/abs/10.3171/2014.V1.FOCUS13451)

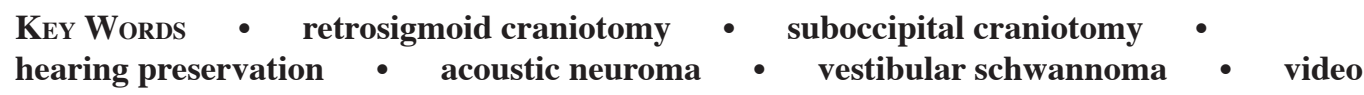

\section{Description of Operative Procedure}

\section{Patient Positioning.}

a. Positioning is an integral component of retrosigmoid craniotomy. Following induction of anesthesia, the patient is moved to the lateral park bench position with rigid head fixation using a three-pin Mayfield skull clamp.

b. All pressure points are padded and an axillary roll is placed. The patient's chest and hips are strapped securely to the table to allow rotation of the table, if necessary. The patient's shoulder is gently retracted inferiorly using tape in order improve access to the surgical field, taking care to avoid excessive traction and resultant brachial plexopathy. The head is rotated approximately 10-15 degrees toward the contralateral side, the neck is flexed slightly and the vertex is tilted slightly down. Special attention is made to ensure that there no pathologic compression of venous drainage from the head (specifically, there should be room for at least two finger breadths between the mandible and upper thorax). Head rotation should not exceed 30 degrees.

c. The body is secured prior to head fixation. Electrophysiologic monitoring (brainstem auditory evoked responses and electromyographic facial nerve monitoring) is then instituted. Longacting paralytics should be avoided as they will interfere with neural integrity monitoring.

Manuscript submitted October 1, 2013.

Accepted November 26, 2013.

Please include this information when citing this paper: DOI: 10.3171/2014.V1.FOCUS13451.

Address correspondence to: Kyle D. Weaver, M.D., Department of Neurological Surgery, 1161 21st Avenue S., Rm. T4224 MCN, Vanderbilt University Medical Center, Nashville, TN 37232-2380. email: Kyle.weaver@vanderbilt.edu.

\section{Surgical Opening Technique}

a. Prior to prepping and draping, external landmarks are assessed. The iniomeatal line is drawn out first with an indelible marker. This dotted line approximates with the level of the transverse sinus. Next, a dotted line is drawn overlying the course of the digastric groove. The portion of this line inferior to the iniomeatal line corresponds to the approximate location of the sigmoid sinus.

b. A curvilinear incision is drawn roughly two-and-a-half fingerbreadths behind the post-auricular sulcus. The patient is the prepped and draped in the usual fashion.

c. Using a scalpel, the incision is made. Dissection is carried down to bone using a scalpel and monopolar cautery. Self-retaining retractors are placed.

$\mathrm{d}$. The asterion is located. With the superficial representation of the junction of the transverse and sigmoid sinuses in mind, a $4 \mathrm{~cm}$ craniotomy is then drawn out posterior and inferior to the sigmoid and transverse sinus respectively. Using a M8 bit to drill a circumferential trough, the bone flap is turned and then elevated.

e. Extradural exposure of the transverse and sigmoid sinuses is then verified. Additional drilling can be performed if exposure is not sufficient. Once the transverse and sigmoid sinuses have been exposed extradurally, the bone edges are waxed thoroughly to decrease the risk of postoperative CSF leak. Any emissary veins are plugged with bone wax or bipolar coagulation. It is important to remove enough bone to allow proper dural reflection and visualization.

f. After meticulous hemostasis has been obtained to obviate run down from the superficial soft tissue, the dura is opened in a curvilinear fashion with a single incision approximately $3-5 \mathrm{~mm}$ away from the margin of the transverse-sigmoid sinus. This dural leaf is covered with a moist rubber dam to prevent shrinkage and is tacked using 4-0 Nurolon.

\section{Dissection to identify existing pathology}

a. After the dura has been reflected and secured, a malleable retractor is placed and CSF is allowed to egress from the foramen 
magnum. When CSF relaxation is deemed adequate, the cerebellum is gently retracted medially and any imposing arachnoid bands are divided in order to gain exposure of the cebellopontine angle.

b. When tumor is visible, the plane involving the posterior margin of tumor is defined.

c. During hearing preservation surgery, the tumor generally has a limited cisternal component $(<1.5 \mathrm{~cm})$ and the internal auditory canal is commonly addressed early on. Careful preoperative review of axial and coronal contrast enhanced T1 weighted MRI and heavy T2 weighted sequences including FIESTA or CISS are important to assess extent of IAC penetration, height and proximity of the jugular bulb in relation to the IAC, and the position of the posterior semicircular canal and vestibule. While we do not routinely obtain a temporal bone CT prior to surgery, it may be beneficial when hearing preservation is attempted.

d. The porus is identified and a leaf of dura at the future site of drilling, over the posterior petrous temporal bone, is bipolar coagulated and resected. Generally, the dural is removed from the just below the tentorium superiorly, just medial to the endolymphatic sac and superior to the pars nervosa, marked by the entry of the lower cranial nerves inferiorly.

e. Next, all cottonoid pledgets are removed from the field and gelfoam is carefully placed in the cistern to help catch any bone dust during drilling. Drilling usually commences with a $3 \mathrm{~mm}$ cutting bur and graduates to a 2 diamond drill when approaching the IAC. Bone is carefully drilled away on a broad front until the lateral internal auditory canal is identified. Next, superior and inferior troughs are developed until the IAC is exposed approximately 270 degrees.

f. From the retrosigmoid vantage, generally only the lateral two-thirds of the IAC can be accessed without violating the posterior semicircular canal or vestibule. The course of the endolymphatic duct may serve as an important landmark to avoid inner ear entry. Additionally, the size of the bur may be used to approximate the extent of lateral drilling. For lateral extending tumors, the posterior canal and vestibule may be "blue lined" for maximum lateral IAC access.

g. After the bone over the IAC has been decompressed, the posterior capsule of the tumor is tested with the flush-tip Prass probe to ensure the facial nerve is following a ventral course. The IAC dura is then opened and the dural leaflets are reflected superiorly and inferiorly. Next, the facial nerve is identified and confirmed using the Prass probe. The tumor is then carefully internally debulked and the capsule is systemically dissected free from the facial and cochlear nerves. Bipolar coagulation is used sparingly and excessive neurovascular traction is avoided since injury to the labyrinthine artery or medial traction on the cochlear nerve may result in hearing loss.

h. Following tumor removal, a 30-degree rigid endoscope may be used to confirm complete resection in tumors involving the lateral IAC. Finally, any perimeatal air cells are occluded with bone wax and a fascia/muscle plug is used to occlude the fundus.

\section{Treatment of the underlying pathological process}

It is important to note that resection of tumor can begin before or after exposure of tumor in the IAC. Prior to resection of the tumor, the posterior surface of the tumor is stimulated with a Prass probe to assess for facial nerve activity. In a small tumor such as this one, an initial attempt can be made to locate the tumor at the brainstem. After the posterior tumor capsule has been verified to be free of facial nerve, the capsule is coagulated and opened. The tumor is debulked using a combination of bipolar electrocautery and tumor forceps. A small sheen of capsule is left and microdissected off of the facial nerve.

\section{Closure}

a. The wound is then irrigated with saline and all retractors are removed. The dura is then closed in a water-tight fashion using interrupted 4-0 Nurolons.

b. Gelfoam is placed on top of the dura, and the bone flap is replaced with KLS screws and plates. Methylmethacrylate is then used to close off the remainder of the cranial opening in order to prevent later insertion of the muscle onto the dura. The wound is then copiously irrigated with Bacitracin irrigation and closed in layers with 2-0 Vicryls in multiple layers in the muscle, 3-0 Vicryls in the galea and dermis, and a running, locked 3-0 nylon in the skin. 